\title{
Aberrant expression of two miRNAs promotes proliferation, hepatitis $b$ virus amplification, migration and invasion of hepatocellular carcinoma cells: evidence from bioinformatic analysis and experimental validation
}

\author{
Yanming Liu ${ }^{1,2}$, Yue Cao ${ }^{3}$, Wencan Cai ${ }^{2}$, Liangyin $\mathrm{Wu}^{2}$, Pingsen Zhao ${ }^{2}$, Xin-guang Liu ${ }^{\text {Corresp. } 1}$ \\ ${ }^{1}$ Guangdong Provincial Key Laboratory of Medical Molecular Diagnostics, Institute of Aging Research, Guangdong Medical University, Dongguan, \\ Guangdong, China \\ 2 Department of Clinical Laboratory, YueBei People's Hospital, Shaoguan, Guangdong, China \\ 3 Department of Medical Technology, Medical College of Shaoguan University, Shaogguan, Guangdong, China \\ Corresponding Author: Xin-guang Liu \\ Email address: ybyylym@gdmu.edu.cn
}

Background. As key negative regulators of gene expression, microRNAs (miRNAs) play an important role in the onset and progression of hepatocellular carcinoma (HCC). This study aimed to identify the miRNAs involved in HCC carcinogenesis and their regulated genes.

Methods. The Gene Expression Omnibus (GEO) dataset (GSE108724) was chosen and explored to identify differentially expressed miRNAs using GEO2R. For the prediction of potential miRNA target genes, the miRTarBase was explored. Enrichment analysis of Gene Ontology (GO) and Kyoto Encyclopedia of Genes and Genomes (KEGG) was performed by the DAVID online tool. The hub genes were screened out using the CytoHubba plug-in ranked by degrees. The networks between miRNAs and hub genes were constructed by Cytoscape software. MiRNA mimics and negative control were transfected into HCC cell lines and their effects on proliferation, hepatitis B virus DNA (HBV-DNA) replication, TP53 expression, migration, and invasion were investigated. The following methods were employed: MTT assay, quantitative PCR (qPCR) assay, western blotting, wound healing assay, and transwell assay.

Results. A total of 50 differentially expressed miRNAs were identified, including 20 upregulated and 30 downregulated miRNAs, in HCC tumor tissues compared to matched adjacent tumor-free tissues. The top three upregulated (miR-221-3p, miR-222-3p, and miR-18-5p) and downregulated (miR-375, miR-214-3p, and miR-378d) miRNAs, ranked by $\mid \log _{2}$ fold change $\left(\log _{2} F C\right) \mid$, were chosen and their potential target genes were predicted. Two gene sets, targeted by the upregulated and the downregulated miRNAs, were identified respectively. GO and KEGG pathway analysis showed that the predicted target genes of upregulated and downregulated miRNAs were mainly enriched in the cell cycle and cancer-related pathways. The top ten hub nodes of gene sets ranked by degrees were identified as hub genes. Analysis of miRNA-hub gene network showed that miR-221-3p and miR-375 modulated most of the hub genes, especially involving regulation of TP53. The q-PCR results showed that miR-221-3p and miR-375 were markedly upregulated and downregulated, respectively, in HCC cells and HCC clinical tissue samples compared to non-tumoral tissues. Furthermore, miR-221-3p overexpression significantly enhanced proliferation, HBV-DNA replication, as well as the migration and invasion of HCC cells, whereas miR-375 overexpression resulted in opposite effects. Western blotting analysis showed that the overexpression of miR-221-3p and miR-375 reduced and increased TP53 expression, respectively. 
Conclusion. The present study revealed that miR-211-3p and miR-375 may exert vital effects on cell proliferation, HBV-DNA replication, cell migration, and invasion through the regulation of TP53 expression in HCC. 


\section{Aberrant expression of two miRNAs promotes}

2 proliferation, hepatitis b virus amplification, migration

3 and invasion of hepatocellular carcinoma cells:

4 evidence from bioinformatic analysis and experimental

5 validation

6

Yanming Liu ${ }^{1,2}$, Yue $\mathrm{Cao}^{3}$, Wencan $\mathrm{Cai}^{2}$, Liangyin $\mathrm{Wu}^{2}$, Pingsen $\mathrm{Zhao}^{2}$, Xinguang Liu ${ }^{1}$

8

${ }^{1}$ Guangdong Provincial Key Laboratory of Medical Molecular Diagnostics, Institute of Aging

Corresponding Author

Xinguang Liu

Songshan lake science and technology park, Dongguan, Guangdong, 523808, China

Email address: ybyylym@gdmu.edu.cn

Research, Guangdong Medical University, Dongguan, Guangdong, China

2 Department of Clinical Laboratory, YueBei People's Hospital, Shaoguan, Guangdong, China

${ }^{3}$ Department of Medical Technology, Medical College of Shaoguan University, Shaoguan, Guangdong, China 
36

37

38

39

40

41

42

43

44

45

46

47

48

\section{Abstract.}

Background. As key negative regulators of gene expression, microRNAs (miRNAs) play an important role in the onset and progression of hepatocellular carcinoma (HCC). This study aimed to identify the miRNAs involved in HCC carcinogenesis and their regulated genes.

Methods. The Gene Expression Omnibus (GEO) dataset (GSE108724) was chosen and explored to identify differentially expressed miRNAs using GEO2R. For the prediction of potential miRNA target genes, the miRTarBase was explored. Enrichment analysis of Gene Ontology (GO) and Kyoto Encyclopedia of Genes and Genomes (KEGG) was performed by the DAVID online tool. The hub genes were screened out using the CytoHubba plug-in ranked by degrees. The networks between miRNAs and hub genes were constructed by Cytoscape software. MiRNA mimics and negative control were transfected into HCC cell lines and their effects on proliferation, hepatitis B virus DNA (HBV-DNA) replication, TP53 expression, migration, and invasion were investigated. The following methods were employed: MTT assay, quantitative PCR (qPCR) assay, western blotting, wound healing assay, and transwell assay.

Results. A total of 50 differentially expressed miRNAs were identified, including 20 upregulated and 30 downregulated miRNAs, in HCC tumor tissues compared to matched adjacent tumor-free tissues. The top three upregulated (miR-221-3p, miR-222-3p, and miR-18-5p) and downregulated (miR-375, miR-214-3p, and miR-378d) miRNAs, ranked by $\mid \log _{2}$ fold change $\left(\log _{2} \mathrm{FC}\right) \mid$, were chosen and their potential target genes were predicted. Two gene sets, targeted by the upregulated and the downregulated miRNAs, were identified respectively. GO and KEGG pathway analysis showed that the predicted target genes of upregulated and downregulated miRNAs were mainly enriched in the cell cycle and cancer-related pathways. The top ten hub nodes of gene sets ranked by degrees were identified as hub genes. Analysis of miRNA-hub gene network showed that miR-221-3p and miR-375 modulated most of the hub genes, especially involving regulation of TP53. The q-PCR results showed that miR-221-3p and miR-375 were markedly upregulated and downregulated, respectively, in HCC cells and HCC clinical tissue samples compared to non-tumoral tissues. Furthermore, miR-221-3p overexpression significantly enhanced proliferation, HBV-DNA replication, as well as the migration and invasion of HCC cells, whereas miR-375 overexpression resulted in opposite effects. Western blotting analysis showed that the overexpression of miR-221-3p and miR-375 reduced and increased TP53 expression, respectively.

Conclusion. The present study revealed that miR-211-3p and miR-375 may exert vital effects on cell proliferation, HBV-DNA replication, cell migration, and invasion through the regulation of TP53 expression in HCC. 


\section{Introduction}

With the second-highest morbidity, third-highest mortality rate, and fifth-highest global incidence in China, hepatocellular carcinoma (HCC) is one of the most lethal malignant tumors, and new cases are increasing worldwide each year (Yanming et al. 2019). The risk factors for HCC are mainly represented by chronic hepatitis B virus (HBV) or hepatitis C virus (HCV) infection, cirrhosis, and alcoholic liver disease (S et al. 2019; X et al. 2019). Alpha-fetoprotein (AFP), the most important tumor marker for HCC diagnosis, has been used for decades but results in a clinically unsatisfactory sensitivity of $40-65 \%$ and a specificity of $76-96 \%$ (Yanming et al. 2019). Therefore, the identification of novel biomarkers of HBV replication and HCC proliferation, migration, and invasion is urgent and crucial for developing effective diagnostic, therapeutic, and prognostic follow-up strategies. MicroRNAs (miRNAs) are endogenous 21-25 nt RNAs that induce mRNA degradation or inhibition of translation by binding to the 3 'untranslated region of target messenger RNAs (mRNAs). It has been demonstrated that the abnormal expression of specific miRNAs promotes the proliferation, invasion, and metastasis of tumor cells (Wang et al. 2018). Therefore, thorough investigation of miRNAs that are functionally related to $\mathrm{HBV}$-infection, as well as to the proliferation, migration, and invasion of HCC cells is of paramount importance.

High-throughput microarray technology has been extensively applied for decades owing to its high sensitivity and processivity. Bioinformatic analysis is widely used to identify differentially expressed miRNAs (DE-miRNAs) and functional pathways involved in carcinogenesis and HCC progression. The combination of these two approaches may help understand the pathogenesis of tumors and improve early diagnosis, treatment, and prognosis of HCC patients (Mantione et al. 2014).

In the present study, the GSE108724 dataset, comprising 7 pairs of HCC and matched adjacent tumor-free tissues, was applied to the screening of 50 differentially expressed miRNAs (DEmiRNAs), including 20 upregulated and 30 downregulated miRNAs related to HCC pathogenesis. The top three upregulated and downregulated miRNAs, ranked by $\mid \log _{2}$ fold change $\left(\log _{2} \mathrm{FC}\right) \mid$, were selected to predict potential target genes, respectively. Enriched Gene Ontology (GO) terms and Kyoto Encyclopedia of Genes and Genomes (KEGG) pathways of each gene set were identified using the DAVID online tool. Protein-protein interaction (PPI) networks were constructed to identify the top ten hub nodes of each gene set, ranked by degrees as hub genes, using the CytoHubba plug-in. Then, the networks established by the top three upregulated and downregulated miRNAs with their 10 target hub genes were constructed. MiR221-3p and miR-375 exhibited the most regulatory relationships with their hub genes, with special regard to TP53 regulation. Subsequently, in vitro validation experiments were performed. As the novelty and innovation of this present research, bioinformatics and experimental validation were combined to explore the effects of miR-211-3p and miR-375 on HBV DNA 
116 amplification, TP53 expression, as well as on the proliferation, migration, and invasion of $\mathrm{HCC}$ 117 cell lines.

\section{Materials and Methods}

120

121

122

123

124

125

126

127

128

129

130

131

132

133

134

135

136

137

138

139

\section{Microarray data}

To explore the role of specific miRNAs in HCC pathogenesis, the GSE108724 dataset (Zhu et al. 2019) was selected and downloaded from GEO (https://www.ncbi.nlm.nih.gov/geo/). This dataset, based on the GPL20712 platform (Agilent-070156 Human miRNA), contained miRNA expression profiles of 7 pairs of HCC and matched adjacent tumor-free tissues.

\section{DE-miRNA identification and target gene prediction}

The DE-miRNAs between HCC and matched adjacent tumor-free tissues were screened using GEO2R (http://www.ncbi.nlm.nih.gov/geo/geo2r) according to the cut-off criterion that $\mathrm{P}$-value $<0.05$ and $\mid \log _{2}$ fold change $\left(\log _{2} \mathbf{F C}\right) \mid>2$, an online interactive web tool with built-in GEOquery and limma R packages that allows to compare two or more datasets across experimental conditions. The GEOquery R package parses GEO data into R data structures that can be used by other $\mathrm{R}$ packages ( $\mathrm{S} \&$ Bioinformatics 2007). The Limma $R$ package is used to homogenize microarray data and to reduce the occurrence of false positives by applying multiple-testing corrections on P-values (ME et al. 2015). The miRTarBase online dataset (http://mirtarbase.mbc.nctu.edu.tw/php/index.php) (version 7.0), containing extensive information on experimentally validated miRNA-target interactions, was utilized for predicting potential miRNA target genes (Chou et al. 2018). Only the top three upregulated and downregulated miRNAs, ranked by $\left|\log _{2} \mathrm{FC}\right|$, were enrolled for target gene prediction analysis and formed two new gene sets of upregulated and downregulated miRNAs, on which GO, KEGG, and hub gene identification analyses were further performed

\section{GO, KEGG, hub gene identification and miRNA-hub gene network construction}

The Database for Annotation, Visualization and Integrated Discovery (DAVID; https://david.ncifcrf.gov) (version 6.8) (Huang da et al. 2009a; Huang da et al. 2009b), providing a comprehensive set of functional annotation information including large lists of genes, allowing for the retrieval of biological information, was applied to perform functional annotation and pathway enrichment analysis for the two gene sets, including GO (Edgar et al. 2002) and KEGG (Kanehisa et al. 2017) pathway analysis. Search Tool for the Retrieval of Interacting Genes (STRING) (https://string-db.org/) is the most popular database of known and predicted protein-protein interactions (PPI). The PPI networks of the two gene sets were constructed using STRING (version 11.0), and interactions with a combined score $>0.4$ were considered statistically significant (Szklarczyk et al. 2019). The top ten hub genes of the two gene sets ranked by degree were selected by using the CytoHubba plug-in (http://apps.cytoscape.org/apps/cytohubba) (version 0.1) (Chin et al. 2014). The Cytoscape software (https://cytoscape.org) (version 3.7.2) was used to construct the networks between three miRNAs and their respective top ten hub target genes (Shannon et al. 2003). P-value $<0.05$ was 
155

156

157

158

159

160

161

162

163

164

165

166

167

168

169

170

171

172

173

174

175

176

177

178

179

180

181

182

183

184

185

186

187

188

189

190

191

192

193

194

195

considered as statistically significant.

Analysis of gene and miRNA expression, survival, and co-expression of miRNAs-hub genes

The Cancer Genome Atlas (TCGA) analysis tool of the UALCAN database

(http://ualcan.path.uab.edu/), an interactive web resource for the analysis of cancer omics data, was utilized to explore differences in the expression of hub genes between normal and tumor tissue in patients with liver hepatocellular carcinoma (LIHC) (Chandrashekar et al. 2017). The OncomiR database (http://www.oncomir.org/), an online resource for the evaluation of cancerrelated miRNA dysregulation, was applied for performing Kaplan-Meier survival analysis based on the expression level of miR-221-3p and miR-375, with 50\% as percentile cutoff value in HCC (Wong et al. 2018). The Pan-Cancer analysis tool of starBase (version 3.0) (http://starbase.sysu.edu.cn/panMirCoExp.php), identifying more than 2.5 million miRNAmRNA interactions from multi-dimensional sequencing data, was used for miRNA-target gene co-expression analysis ( $\mathrm{Li}$ et al. 2014). P-value of $<0.05$ was considered as statistically significant.

\section{HCC cell lines, clinical samples, and cell transfection}

Immortalized normal human liver HL7702 cells and the HCC cell lines HepG2, HepG2.2.15, and MHCC-LM3 were purchased from CELLCOOK CO. LTD (Guangzhou, China). All cells were cultured in Dulbecco's modified Eagle's medium/High Glucose (DMEM; 8885329, Gibco, Shanghai, China) with 10\% fetal bovine serum (FBS; 11011-8611, TIANHANG, Hangzhou, China) and maintained under a humidified atmosphere of $5 \% \mathrm{CO} 2$ at $37{ }^{\circ} \mathrm{C}$. With the written informed consent from participants and the approval obtained from the institutional ethics review board of YueBei people's Hospital (approval number: SUMC-IRB-2017), HCC tumor tissues and matched adjacent tumor-free tissues were obtained from YueBei People's Hospital.

According to the manufacturer's instructions, the riboFECT CP Transfection Kit (C10511-05, RiboBio, Guangzhou, China) was used to transfect miRNA mimics and negative control (RiboBio, Guangzhou, China) into HCC cell lines.

\section{RNA isolation and quantitative reverse transcription PCR(qRT-PCR)}

Total RNA from different cell lines and human tissues was extracted using the RNA Extraction Kit (R1405; GBCBIO, Guangzhou, China). The extracted RNA was stored at $-80{ }^{\circ} \mathrm{C}$ after measuring the concentration and quality. Stem-loop reverse transcriptase (RT) primers for two miRNAs were designed and purchased from TSINGKE Biological Technology Co., Ltd., Beijing, China. Q-PCR was performed three times using SYBR Premix Ex Taq (Q111-02; Vazyme, Nanjing, China) and normalized with U6 small nuclear RNA as internal reference. The $2-\Delta \Delta C T$ method was applied to determine the relative expression level of miRNAs. The sequences of qRT-PCR primers are shown in Table S1 of the Supplementary file.

\section{MTT cell proliferation assay}

HepG2 cells $\left(2 \times 10^{3}\right.$ cells/well) were plated in 96-well plates. After transfection (final transfection concentration of miRNA mimics and negative control: $50 \mathrm{nM}$ ) and growth to $95 \%$ confluence, HepG2 cells were harvested. Cell proliferation was analyzed in triplicate by using the MTT assay kit (G7420-2, GBCBIO, Guangzhou, China) according to the manufacturer's instruction. 
196

197

198

199

200

201

202

203

204

205

206

207

208

209

210

211

212

213

214

215

216

217

218

219

220

221

222

223

224

225

226

227

228

229

230

231

232

233

234

235

\section{HBV-DNA qPCR detection}

HepG2.2.15 cells $\left(1 \times 10^{5}\right.$ cells/well $)$ were seeded in 6-well plates. After transfection, the HBVDNA quantitative kit (2019005, DAAN Gene, Guangzhou, China), including the reagents for DNA extraction, PCR amplification, calibration, and quality control, was used for measuring the level of HBV-DNA in $1 \mathrm{~mL}$ of cell culture supernatant, as per manufacturer's instructions.

\section{Western blot}

Western blot was performed as previously described with minor modifications (biology 2016). HepG2 cells $\left(1 \times 10^{5}\right.$ cells/well $)$ were seeded in 6-well plates. After transfection and growth to $95 \%$ confluence, the cells were harvested. The following primary antibodies were used: anti-p53 (ab131442, Abcam, Cambridge, UK, 1:100) and anti-GAPDH (EPR16891, Abcam, Cambridge, UK, 1:100). The horseradish peroxidase-labeled secondary antibody ab205718, Abcam, Cambridge, UK was used (1:500). A DAB Horseradish Peroxidase Color Development Kit (G3433; GBCBIO, Guangzhou, China) was employed to visualize p53 and GAPDH. The colored bands on the PVDF membrane were photographed with an OLYMPUS microscope (Tokyo, Japan) and permanently stored.

\section{Wound healing assays}

HepG2 cells $\left(1 \times 10^{5}\right.$ cells/well $)$ were seeded in 12 -well plates. After transfection and growth to $95 \%$ confluence, a sterilized pipette tip was used to scratch a wound. The wounded areas were photographed with a microscope, immediately and after $24 \mathrm{~h}$ (magnification: 100×).

\section{Transwell assay}

The cell invasion assay was performed using 24-well transwell chambers (3422, Corning, USA).

The transwell chamber (upper compartment) was separated from the 24-well plate (lower compartment) by polycarbonate membrane with a pore size of $8 \mu \mathrm{m}$. The upper surface of the polycarbonate membrane was coated with Matrigel (354230, BD Bioscience, USA, 1:8 dilution). After transfection, $200 \mu \mathrm{L}$ of serum-free medium containing $1 \times 10^{5} \mathrm{HepG} 2$ cells were added to the upper compartment. Next, $600 \mu \mathrm{L}$ of medium containing $20 \%$ FBS were added to the lower compartment. Then, after a $24-\mathrm{h}$ incubation at $37^{\circ} \mathrm{C}$ and removal of the cells on the upper surface of the membrane in the upper compartment using a cotton bud, the membranes were fixed (95\% methanol) for $30 \mathrm{~min}$, stained (0.1\% crystal violet) for $20 \mathrm{~min}$ at room temperature, and three visual fields were photographed (magnification: $200 \times$ ) under a microscope (OLYMPUS, Tokyo, Japan). The experiments were independently repeated in triplicate.

\section{Statistical analysis}

The results were expressed as mean $\pm \mathrm{SD}$. The raw data obtained with the HBV-DNA assay were subjected to base 10 logarithmic conversion before statistical analysis. One-way ANOVA analysis was used to compare the miRNA expression between four cell lines. The twoindependent $t$-test was used to compare miRNAs expression between para-tumor and tumor tissues. Unless otherwise noted, statistical diagrams were drawn using GraphPad Prism (version 8.0.1, GraphPad Software, USA). For all analyses, $\mathrm{P}<0.05$ was regarded as statistically significant. 


\section{Results}

\section{Identification of DE-miRNAs and of their potential target genes in HCC} The GEO2R online tool was applied to screen 50 DE-miRNAs between 7 HCC tissues and their matched adjacent tumor-free tissues in the GSE108724 dataset (P-value $<0.05$ and $\left|\log _{2} \mathrm{FC}\right|>2$ ). Twenty upregulated and thirty downregulated DE-miRNAs are shown in the volcano plot (Fig. 1). Table 1 and Table 2 show the top ten upregulated and downregulated DE-miRNAs, respectively, ranked by $\left|\log _{2} \mathrm{FC}\right|$. A total of 741 potential target genes were predicted for the top three upregulated miRNAs (miR-221-3p, and miR-222-3p, and miR-18b-5p), while 696 genes were identified as candidate targets of the top three downregulated miRNAs (miR-375, miR-214$3 p$, and miR-378d) by using the miRTarBase online tool.

\section{Functional enrichment analysis of target genes}

To comprehensively investigate the biological functions of target genes, GO and KEGG pathway enrichment analyses were carried out using the DAVID online tool. GO analysis can be divided into three functional groups: biological process group (BP), molecular function group (MF), and cellular component group (CC). GO analysis of the 741 potential target genes of the three upregulated miRNAs revealed that changes in BP were mainly enriched in negative regulation of transcription from RNA polymerase II promoter, positive regulation of transcription, and cell-cell adhesion (Fig. S1A); changes in MF were mainly enriched in poly(A) RNA binding, protein binding, and cadherin binding involved in cell-cell adhesion (Fig. S1C); changes in CC were mainly enriched in nucleoplasm, nucleus, and cytosol (Fig. S1E). GO analysis of the 696 target genes of the three downregulated miRNAs revealed that changes in BP were mainly involved positive regulation of neuron apoptotic process, organ morphogenesis, and blood vessel remodeling (Fig. S1B); changes in MF were mainly involved GDP binding, transcription coactivator activity, and mRNA binding (Fig. S1D); changes in CC were mainly invloved nucleus, extracellular exosome, and nucleoplasm (Fig. S1F). KEGG pathway analysis showed that the 741 candidate target genes of the three upregulated miRNAs were mainly enriched in cell cycle, cancer-related pathways, and FoxO signaling pathway (Fig.S1G); the 696 target genes of the three downregulated miRNAs were mainly involved cancer-related pathways, breast cancer, hepatitis B, and MAPK signaling pathway (Fig. S1H).

\section{Hub gene identification, miRNA-hub gene network construction, and targeted gene expression analysis in HCC tissues}

The top 10 nodes ranked by degrees of the CytoHubba plug-in of Cytoscape software were recognized as hub genes. Six (TP53, MYC, HSP90AA1, PTEN, CASP3, CTNNB1) of ten hub genes were in common between upregulated and downregulated DE-miRNAs, albeit they exhibited different scores (Table 3). TP53, exhibiting the highest score among upregulated and downregulated DE-miRNAs, could play a vital role in HCC carcinogenesis or progression. The 
272

273

274

275

276

277

278

279

280

281

282

283

284

285

286

287

288

289

290

291

292

293

294

295

296

297

298

299

300

301

302

303

304

305

306

307

308

309

310

analysis of the miRNA-Hub gene network revealed that miR-221-3p potentially modulated seven (PTEN, TP53, ACTB, ESR1, CTNNB1, CASP3, and UBC) of ten hub genes. Moreover, miR-222$3 \mathrm{p}$ and $\mathrm{miR}-18 \mathrm{~b}-5 \mathrm{p}$ regulated six and two hub genes, respectively (Fig. 2A). Interestingly, miR375 potentially modulated five (HSP90AA1, CASP3, MYC, TP53, and CTNNB1) of 10 hub genes. MiR-214-3p and miR-378d could regulate three and zero hub genes, respectively (Fig. 2B). Eleven significantly upregulated hub genes (UBC, ACTB, TP53, HSP90AA1, PTEN, $C A S P 3, C T N N B 1, A K T 1, M A P K 3, C D C 42$, and MAPK1) (Fig.3A, D, E, I-P)and two significantly downregulated hub genes (ESRl and MYC) (Fig.3C, H) were compared in HCC tissues and normal liver tissues using the TCGA analysis tool of the UALCAN database. The results of TCGA analysis showed that the expression level of TP53 was positively related to HCC tumor stage and metastasis. (Fig.3F-G). TP53, one of the hub genes in common between the two sets of DE-miRNAs, exhibiting the highest score, maybe the most potentially hub gene of miR-221-3p and miR-375. Based on the above findings, we speculated that miR-221-3p and miR-375 might play critical roles in HCC by regulating TP53 expression.

\section{The expression level of miR-221-3p and miR-375 in HCC cells and clinical samples} Three HCC cell lines (HepG2, HepG2.2.15, and HCC-LM3) and a normal liver cell line (HL7702) were employed to evaluate the expression level of miR-221-3p and miR-375. qRTPCR revealed that the two miRNAs were significantly upregulated and downregulated in all three HCC cell lines, respectively, compared to HL7702 cells (Fig.4 A, D). Notably, the expression levels of miR-221-3p and miR-375 were the highest and lowest in HCC-LM3 compared to the other two HCC cell lines, respectively. Due to the high invasion capacity of HCC-LM3 cells (Zha et al. 2018), this suggested that miR-221-3p and miR-375 could be critical regulators of HCC cell invasion. HepG2.2.15 is an HCC cell line that carries and secretes HBV particles. Notably, in these cells, miR-221-3p expression was much higher, and that of miR-375 much lower, compared to HepG2 cells. These findings were consistent with an important role of miR-221-3p and miR-375 in the tumorigenesis of HBV-related HCC. Subsequently, qRT-PCR analysis of HCC tissue samples demonstrated that miR-221-3p and miR-375 were, respectively, upregulated and downregulated in $20 \mathrm{HCC}$ tissue samples compared to their para-tumor tissues (Fig. 4B, E). Next, the OncomiR online database was employed to further evaluate the expression level and the prognostic value of miR-221-3p and miR-375 in HCC patients. The mean expression level of miR-221-3p was much higher, and that of miR-375 much lower, in HCC tissues compared to normal liver tissues (Fig. 4 C, F). Interestingly, high miR-221-3p expression and low miR-375 expression tended to be correlated with poor overall survival. However, this association was not statistically significant $(\mathrm{P}=0.06034$ for miR-221-3p and $\mathrm{P}=0.3215$ for miR-375) (Fig. $4 \mathrm{G}-\mathrm{H}$ ). In conclusion, although further experimental validation is needed, miR-221-3p and miR-375 are potential prognostic biomarkers, with special regard to HBV amplification and HCC invasion.

The effects of miR-221-3p and miR-375 overexpression on proliferation, HBV-DNA replication, migration, and invasion of $\mathrm{HCC}$ cells 
311 HepG2 cells were transfected with $50 \mathrm{nM}$ or $100 \mathrm{nM}$ miRNA mimics. qRT-PCR analysis

312 showed that miRNA expression increased with the concentration of transfected miRNA mimics

313 (Fig.5 A). After experimental verification, $50 \mathrm{nM}$ was selected as the most suitable concentration 314 of the two miRNAs for subsequent transfections. First, we performed an MTT assay to explore 315 the impact of miR-221-3p and miR-375 on HCC cell proliferation. The results showed that miR316 221-3p and miR-375 overexpression significantly enhanced and decreased, respectively, HepG2 317 cell proliferation when compared to HepG2 cells transfected with the negative control (Fig.5 B).

318 In addition, we performed an HBV-DNA qPCR assay to determine the effect of miR-221-3p and 319 miR-375 overexpression on the replication of HBV-DNA. The qPCR results showed that the 320 upregulation of miR-221-3p and miR-375 increased and decreased, respectively, the level of 321 HBV-DNA in HepG2.2.15 cells (Fig. 5 C). Moreover, western blot analysis indicated that miR322 221-3p and miR-375 overexpression weakened and enhanced, respectively, the expression of 323 TP53 (Fig.5 D). The co-expression experiments showed that the level of TP53 expression was value: 7.16E-10; miR-375: r=0.036, P-value: 4.59E-1) (Fig.5 E-F). Moreover, we performed a wound healing assay to evaluate the role of miR-221-3p and miR-375 in HCC cell migration. HepG2 cell migration was positively correlated with miR-221-3p overexpression and negatively correlated with miR-375 overexpression (Fig.6 A-B). Finally, the effects of the two miRNAs on HCC cell invasion were verified by a transwell assay, which demonstrated that miR-221-3p and miR-375 overexpression enhanced and inhibited, respectively, HepG2 cell invasion (Fig.6 C-D). In summary, the data confirmed that miR-221-3p and miR-375 are potential regulators of TP53 expression, and could therefore affect HBV-DNA replication, as well as HCC cell proliferation, migration, and invasion.

\section{Discussion}

The pathogenesis $\mathrm{HCC}$ is still unclear, and the known etiological factors mainly include exogenous factors, such as hepatitis virus infection, and endogenous factors, such as excessive drinking, metabolic disorders, and environmental factors (Kokudo et al. 2019). The 5-year survival of HCC patients can be higher than 50\% after early diagnosis (Forner et al. 2012). However, due to delayed diagnosis, most HCC patients are not suitable for further treatment and often have a poor prognosis.

Numerous studies had demonstrated that miRNAs play an important role in various biological processes of HCC cells, such as cell proliferation, HBV persistent infection, tumor suppressor genes expression, and metastasis. In China, the persistent exacerbation of HBV amplification is the main determinant of HCC recurrence (Yanming et al. 2019). A recent study showed that miR-146a SNP $(\mathrm{C} / \mathrm{G})$ is associated with the susceptibility to HBV infection and with spontaneous viral clearance (Khanizadeh et al. 2019). TP53 mutation, the most common mutation in HCC, affects the progression and prognosis of HCC (Long et al. 2019). Interestingly, miR-487a stimulates cancer cell proliferation via PIK3R1-mediated AKT signaling and promotes metastasis via SPRED2-induced mitogen-activated protein kinase signaling in HCC (Chang et al. 
351

352

353

354

355

356

357

358

359

360

361

362

363

364

365

366

367

368

369

370

371

372

373

374

375

376

377

378

379

380

381

382

383

384

385

386

387

388

389

390

391

2017). Hence, it is urgent need to systematically analyzed and experimentally verified the role of specific miRNAs in HBV amplification, as well as in the proliferation, migration, and invasion of HCC cells.

In this study, a data-driven decision was aaplied to gradually narrow down the scope of experimental verification miRNAs (W et al. 2018). First, we screened 20 upregulated and 30 downregulated miRNAs on the basis of the miRNA expression profiles of the GSE108724 dataset. Subsequently, potential miRNA target genes were predicted for the top three upregulated and downregulated miRNAs ranked by $\left|\log _{2} \mathrm{FC}\right|$. According to the results of GO and KEGG analysis, the identified miRNAs were implicated in focal adhesion, cell cycle regulation, HBV infection, and mitosis. Next, the top ten hub genes, ranked by degrees, of upregulated and downregulated miRNAs were identified. The generated miRNA-hub gene network showed that miR-221-3p and miR-375 modulated most of the hub genes. Moreover, the two latter miRNAs exhibited the highest score regarding TP53 regulation. However, as far as we know, the effect of TP53 expression modulated by miR-221-3p and miR-375 on the proliferation, invasion, metastasis, and HBV replication in HCC have rare been reported. It is of great significance to clarify the mechanism how the two miRNAs regulate the above phenotypes of HCC.

The present study confirmed that the expression of miR-221-3p and miR-375 was significantly upregulated and downregulated, respectively, in both HCC cell lines and HCC tissue samples. The upregulation of miR-212-3p and miR-375 enhanced and inhibited, respectively, HBV-DNA replication and amplification, as well as HCC cell migration and invasion. In addition, the upregulation of miR-212-3p and miR-375 exerted opposite effects on TP53 expression, respectively suppressing and promoting its transcription. Recent studies have demonstrated that miR-221-3p functions as an oncogene in specific types of human cancer, including HCC (Wang et al. 2019), non-small cell lung cancer(Yin et al. 2019), glioma (Milani et al. 2019), gastric cancer (Zhang et al. 2019)and breast cancer (Deng et al. 2017). It was also reported that miR-375 inhibits the proliferation and migration of many cancer cells (Guo et al. 2018; Shen et al. 2014; Wu et al. 2017). Moreover, miR-221 promotes HCC cell migration by regulating the expression of plant homeodomain finger 2 (PHF2), which was demonstrated to be a target gene of miR-221 (Y et al. 2019). Furthermore, PHF2 acts as a vital regulator of cancer development in association with TP53, and is required for TP53-mediated cell death ( $\mathrm{KH}$ et al. 2015). Several studies demonstrated that miR-375 negatively regulates epithelial-mesenchymal transition, apoptosis, and cell migration by directly blocking its target, YWHAZ or ErbB2 in HCC cells (Li et al. 2018; Zhao et al. 2018). A potent oncogenic cooperation between mutant TP53 and ErbB2 was also demonstrated in human Her2-positive breast cancer (D \& Oncotarget 2017). On the basis of our findings, we reasoned that, in association with miR-221-3p and miR-375, TP53 could act as a tumor suppressor by inhibiting HBV replication, proliferation, migration, and invasion of HCC cells, and promote p53-mediated cell apoptosis.

Unfortunately, there are some limitations in this study. First, despite the fact that the unvalidated microRNAs had a similar numbers of targets to the validated microRNAs, only the two miRNAs with significant variation were selected and further analyzed. Moreover, a dualluciferase reporter experiment was not performed to directly verify whether TP53 was the target 
392

393

394

395

396

397

398

399

400

401

402

403

404

405

406

407

408

409

410

411

412

413

414

415

416

417

418

419

420

421

422

423

424

425

426

427

428

429

gene of miR-221-3p and miR-375. The latter aspect will be the focus of forthcoming research in our laboratory.

\section{Conclusions}

In sum, we demonstrated that the aberrant expression of miR-221-3p and miR-375 may affect HBV-DNA replication, as well as the proliferation, migration, and invasion of HCC cells, possibly by modulating the expression of TP53. Thus, these two miRNAs may serve as crucial biomarkers for HCC diagnosis and prognosis.

\section{Acknowledgements}

I would like to thank professor Xing Guang Liu for his valuable suggestions on the design of the present study.

\section{Reference}

biology MPJMim. 2016. Preparation of Cell Lysate from Mouse Oocytes for Western Blotting Analysis. 1457:209215. 10.1007/978-1-4939-3795-0_15

Chandrashekar DS, Bashel B, Balasubramanya SAH, Creighton CJ, Ponce-Rodriguez I, Chakravarthi B, and Varambally S. 2017. UALCAN: A Portal for Facilitating Tumor Subgroup Gene Expression and Survival Analyses. Neoplasia 19:649-658. 10.1016/j.neo.2017.05.002

Chang RM, Xiao S, Lei X, Yang H, Fang F, and Yang LY. 2017. miRNA-487a Promotes Proliferation and Metastasis in Hepatocellular Carcinoma. Clin Cancer Res 23:2593-2604. 10.1158/1078-0432.Ccr-16-0851

Chin $\mathrm{CH}$, Chen SH, Wu HH, Ho CW, Ko MT, and Lin CY. 2014. cytoHubba: identifying hub objects and sub-networks from complex interactome. BMC Syst Biol 8:S11. 10.1186/1752-0509-8-s4-s11

Chou CH, Shrestha S, Yang CD, Chang NW, Lin YL, Liao KW, Huang WC, Sun TH, Tu SJ, Lee WH, Chiew MY, Tai CS, Wei TY, Tsai TR, Huang HT, Wang CY, Wu HY, Ho SY, Chen PR, Chuang CH, Hsieh PJ, Wu YS, Chen WL, Li MJ, Wu YC, Huang XY, Ng FL, Buddhakosai W, Huang PC, Lan KC, Huang CY, Weng SL, Cheng YN, Liang C, Hsu WL, and Huang HD. 2018. miRTarBase update 2018: a resource for experimentally validated microRNAtarget interactions. Nucleic Acids Res 46:D296-D302. 10.1093/nar/gkx1067

D L, and Oncotarget MNJ. 2017. ErbB2 inhibition by lapatinib promotes degradation of mutant p53 protein in cancer cells. 8:5823-5833. 10.18632/oncotarget.12878

Deng L, Lei Q, Wang Y, Wang Z, Xie G, Zhong X, Wang Y, Chen N, Qiu Y, Pu T, Bu H, and Zheng H. 2017. Downregulation of miR-221-3p and upregulation of its target gene PARP1 are prognostic biomarkers for triple negative breast cancer patients and associated with poor prognosis. Oncotarget 8:108712-108725. 10.18632/oncotarget.21561

Edgar R, Domrachev M, and Lash AE. 2002. Gene Expression Omnibus: NCBI gene expression and hybridization array data repository. Nucleic Acids Res 30:207-210. 10.1093/nar/30.1.207

Forner A, Llovet JM, and Bruix J. 2012. Hepatocellular carcinoma. Lancet 379:1245-1255. 10.1016/s01406736(11)61347-0

Guo J, Liu X, Yang Y, Liang M, Bai C, Zhao Z, and Sun B. 2018. miR-375 down-regulation of the rearranged L-myc 
430

431

432

433

434

435

436

437

438

439

440

441

442

443

444

445

446

447

448

449

450

451

452

453

454

455

456

457

458

459

460

461

462

463

464

465

466

467

468

469

470

fusion and hypoxia-induced gene domain protein $1 \mathrm{~A}$ genes and effects on Sertoli cell proliferation. AsianAustralas J Anim Sci 31:1103-1109. 10.5713/ajas.17.0338

Huang da W, Sherman BT, and Lempicki RA. 2009a. Bioinformatics enrichment tools: paths toward the comprehensive functional analysis of large gene lists. Nucleic Acids Res 37:1-13. 10.1093/nar/gkn923

Huang da W, Sherman BT, and Lempicki RA. 2009b. Systematic and integrative analysis of large gene lists using DAVID bioinformatics resources. Nat Protoc 4:44-57. 10.1038/nprot.2008.211

Kanehisa M, Furumichi M, Tanabe M, Sato Y, and Morishima K. 2017. KEGG: new perspectives on genomes, pathways, diseases and drugs. Nucleic Acids Res 45:D353-D361. 10.1093/nar/gkw1092

KH L, JW P, HS S, YJ C, WH K, HS L, HJ C, HW S, CH C, TY K, SH L, HD Y, SJ K, and Oncogene CYJ. 2015. PHF2 histone demethylase acts as a tumor suppressor in association with p53 in cancer. 34:2897-2909. 10.1038/onc.2014.219

Khanizadeh S, Hasanvand B, Nikoo HR, Anbari K, Adhikary H, Shirkhani S, and Lashgarian HE. 2019. Association between miRNA-146a rs2910164 (G/C) polymorphism with the susceptibility to chronic HBV infection and spontaneous viral clearance in an Iranian population. J Med Virol 91:1063-1068. 10.1002/jmv.25394

Kokudo N, Takemura N, Hasegawa K, Takayama T, Kubo S, Shimada M, Nagano H, Hatano E, Izumi N, Kaneko S, Kudo M, lijima H, Genda T, Tateishi R, Torimura T, Igaki H, Kobayashi S, Sakurai H, Murakami T, Watadani T, and Matsuyama Y. 2019. Clinical practice guidelines for hepatocellular carcinoma: The Japan Society of Hepatology 2017 (4th JSH-HCC guidelines) 2019 update. Hepatol Res. 10.1111/hepr.13411

Li JH, Liu S, Zhou H, Qu LH, and Yang JH. 2014. starBase v2.0: decoding miRNA-ceRNA, miRNA-ncRNA and proteinRNA interaction networks from large-scale CLIP-Seq data. Nucleic Acids Res 42:D92-97. 10.1093/nar/gkt1248

Li L, Jia L, and Ding Y. 2018. Upregulation of miR-375 inhibits human liver cancer cell growth by modulating cell proliferation and apoptosis via targeting ErbB2. Oncol Lett 16:3319-3326. 10.3892/ol.2018.9011

Long J, Wang A, Bai Y, Lin J, Yang X, Wang D, Yang X, Jiang Y, and Zhao H. 2019. Development and validation of a TP53-associated immune prognostic model for hepatocellular carcinoma. EBioMedicine 42:363-374. 10.1016/j.ebiom.2019.03.022

Mantione KJ, Kream RM, Kuzelova H, Ptacek R, Raboch J, Samuel JM, and Stefano GB. 2014. Comparing bioinformatic gene expression profiling methods: microarray and RNA-Seq. Med Sci Monit Basic Res 20:138-142. 10.12659/msmbr.892101

ME R, B P, D W, Y H, CW L, W S, and research SGJNa. 2015. limma powers differential expression analyses for RNAsequencing and microarray studies. 43:e47. 10.1093/nar/gkv007

Milani R, Brognara E, Fabbri E, Manicardi A, Corradini R, Finotti A, Gasparello J, Borgatti M, Cosenza LC, Lampronti I, Dechecchi MC, Cabrini G, and Gambari R. 2019. Targeting miR-155-5p and miR-221-3p by peptide nucleic acids induces caspase-3 activation and apoptosis in temozolomide-resistant T98G glioma cells. Int J Oncol 55:59-68. 10.3892/ijo.2019.4810

S D, and Bioinformatics MPJ. 2007. GEOquery: a bridge between the Gene Expression Omnibus (GEO) and BioConductor. 23:1846-1847. 10.1093/bioinformatics/btm254

S X, X J, J Z, S X, Y H, R W, and PeerJ YYJ. 2019. Identification of significant gene and pathways involved in HBVrelated hepatocellular carcinoma by bioinformatics analysis. 7:e7408. 10.7717/peerj.7408

Shannon P, Markiel A, Ozier O, Baliga NS, Wang JT, Ramage D, Amin N, Schwikowski B, and Ideker T. 2003. Cytoscape: a software environment for integrated models of biomolecular interaction networks. Genome

Peer) reviewing PDF | (2019:12:43700:2:0:NEW 4 Apr 2020) 
Res 13:2498-2504. 10.1101/gr.1239303

Shen ZY, Zhang ZZ, Liu H, Zhao EH, and Cao H. 2014. miR-375 inhibits the proliferation of gastric cancer cells by repressing ERBB2 expression. Exp Ther Med 7:1757-1761. 10.3892/etm.2014.1627

Szklarczyk D, Gable AL, Lyon D, Junge A, Wyder S, Huerta-Cepas J, Simonovic M, Doncheva NT, Morris JH, Bork P, Jensen L, and Mering CV. 2019. STRING v11: protein-protein association networks with increased coverage, supporting functional discovery in genome-wide experimental datasets. Nucleic Acids Res 47:D607-D613. 10.1093/nar/gky1131

W L, J C, B D, D C, H Z, D J, L X, C B, G C, and medicine FWJJot. 2018. Identification of invasion-metastasisassociated microRNAs in hepatocellular carcinoma based on bioinformatic analysis and experimental validation. 16:266. 10.1186/s12967-018-1639-8

Wang T, Xu H, Qi M, Yan S, and Tian X. 2018. miRNA dysregulation and the risk of metastasis and invasion in papillary thyroid cancer: a systematic review and meta-analysis. Oncotarget 9:5473-5479. 10.18632/oncotarget.16681

Wang X, Liao X, Huang K, Zeng X, Liu Z, Zhou X, Yu T, Yang C, Yu L, Wang Q, Han C, Zhu G, Ye X, and Peng T. 2019. Clustered microRNAs hsa-miR-221-3p/hsa-miR-222-3p and their targeted genes might be prognostic predictors for hepatocellular carcinoma. J Cancer 10:2520-2533. 10.7150/jca.29207

Wong NW, Chen Y, Chen S, and Wang X. 2018. OncomiR: an online resource for exploring pan-cancer microRNA dysregulation. Bioinformatics 34:713-715. 10.1093/bioinformatics/btx627

Wu Y, Sun X, Song B, Qiu X, and Zhao J. 2017. MiR - 375/SLC7A11 axis regulates oral squamous cell carcinoma proliferation and invasion. Cancer Med 6:1686-1697. 10.1002/cam4.1110

X Z, C K, N L, X L, J Z, F G, and PeerJ DL. 2019. Identification of special key genes for alcohol-related hepatocellular carcinoma through bioinformatic analysis. 7:e6375. 10.7717/peerj.6375

Y F, M L, F L, L Q, P Z, F L, W C, and international HRJBr. 2019. MiR-221 Promotes Hepatocellular Carcinoma Cells Migration via Targeting PHF2. 2019:4371405. 10.1155/2019/4371405

Yanming L, Yue C, Wencan C, Liangyin WU, Xijun L, Jiafeng Z, and Fengping HE. 2019. Combined detection of AFPL3, GP73 and TIP30 enhances diagnostic accuracy for HBV-related cirrhosis and hepatocellular carcinoma. J Pak Med Assoc 69:1279-1286.

Yin G, Zhang B, and Li J. 2019. miR-221-3p promotes the cell growth of non-small cell lung cancer by targeting p27. Mol Med Rep 20:604-612. 10.3892/mmr.2019.10291

Zha Y, Yao Q, Liu JS, Wang YY, and Sun WM. 2018. Hepatitis B virus X protein promotes epithelial-mesenchymal transition and metastasis in hepatocellular carcinoma cell line HCCLM3 by targeting HMGA2. Oncol Lett 16:5709-5714. 10.3892/ol.2018.9359

Zhang Y, Huang H, Zhang Y, and Liao N. 2019. Combined Detection of Serum MiR-221-3p and MiR-122-5p Expression in Diagnosis and Prognosis of Gastric Cancer. J Gastric Cancer 19:315-328. 10.5230/jgc.2019.19.e28

Zhao JF, Zhao Q, Hu H, Liao JZ, Lin JS, Xia C, Chang Y, Liu J, Guo AY, and He XX. 2018. The ASH1-miR-375-YWHAZ Signaling Axis Regulates Tumor Properties in Hepatocellular Carcinoma. Mol Ther Nucleic Acids 11:538553. 10.1016/j.omtn.2018.04.007

Zhu HR, Yu XN, Zhang GC, Shi X, Bilegsaikhan E, Guo HY, Liu LL, Cai Y, Song GQ, Liu TT, Dong L, Janssen HLA, Weng SQ, Wu J, Shen XZ, and Zhu JM. 2019. Comprehensive analysis of long noncoding RNAmessenger RNAmicroRNA coexpression network identifies cell cyclerelated IncRNA in hepatocellular carcinoma. Int J 
513

514 


\section{Figure 1}

Figure 1 Volcano plot of DE-miRNAs between 7 pairs of $\mathrm{HCC}$ and matched adjacent tumor-free tissues in the GSE108724dataset.

Red and green dots represent upregulated and downregulated miRNAs, respectively, based on p-value $<0.05$ and $\left|\log _{2} \mathrm{FC}\right|>2$; black dots represent miRNAs with no significant expression differences. 


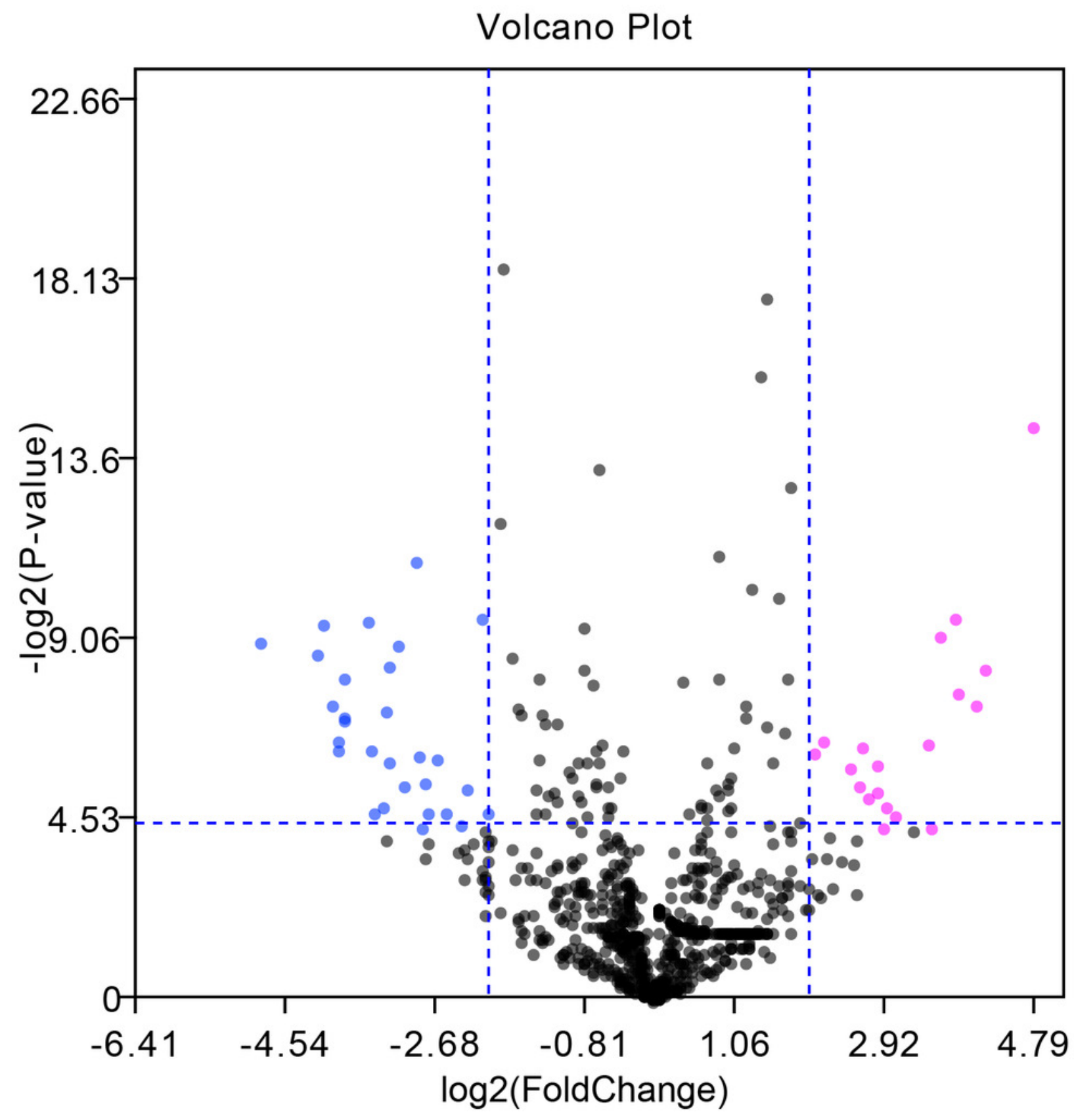




\section{Figure 2}

Figure 2 The miRNA-hub gene network was constructed using Cytoscape software.

(A) Network of three upregulated miRNAs and their predicted hub genes. (B) Network of three downregulated miRNAs and their predicted hub genes. Red diamonds represent upregulated miRNAs, green diamonds represent downregulated miRNAs, cyan rectangles represent hub genes, lines represent the interaction between miRNAs and hub genes, and free diamonds and rectangle represent the absence of interaction between miRNAs and hub genes.

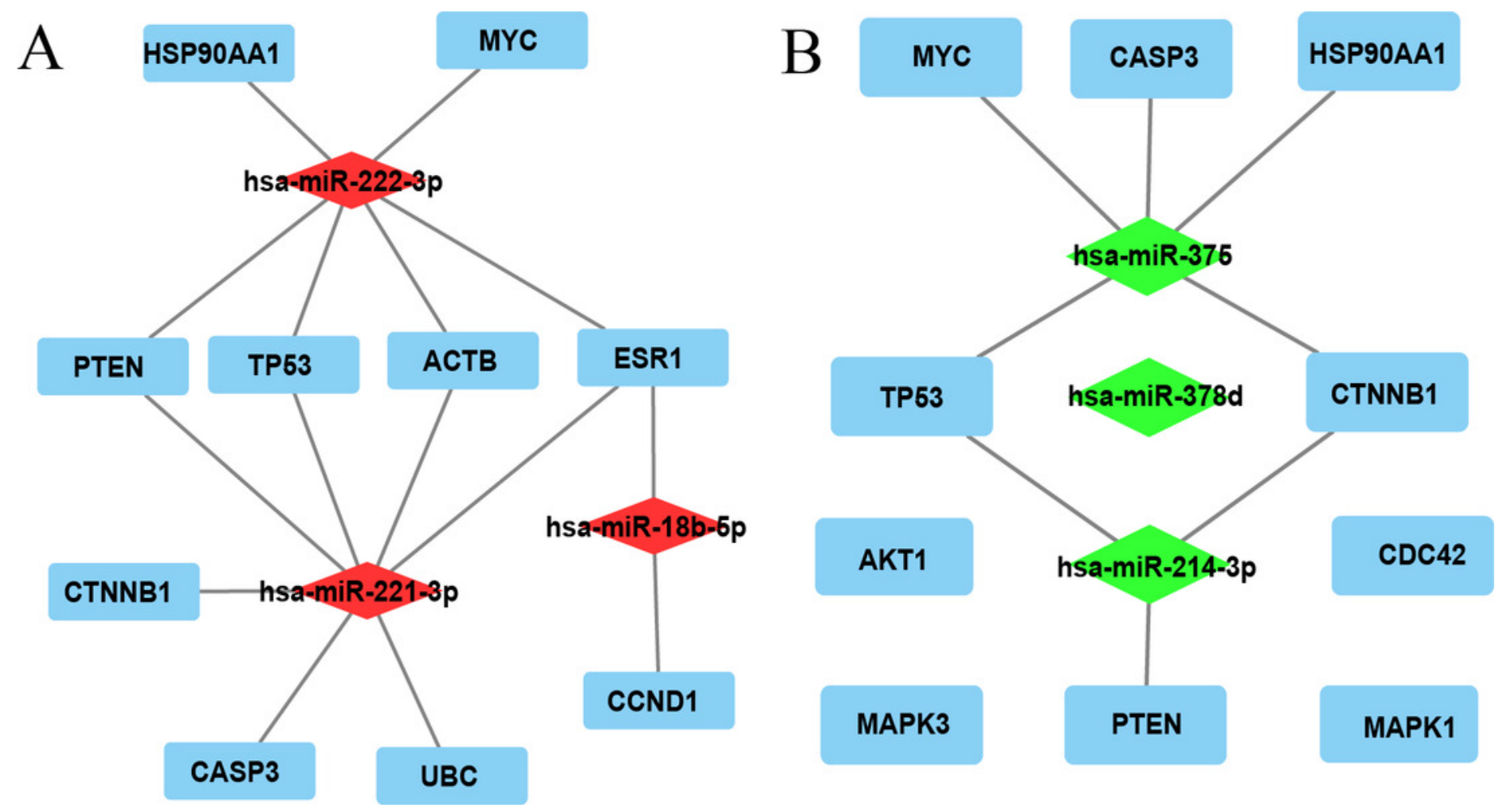




\section{Figure 3}

Figure 3 mRNA expression of the predicted hub genes of miR-221-3p and miR-375 from the UALCAN LIHC database.

The hub genes were potential predictive targets of miR-211-3p (A-L) and miR-375 (E-P). (A) UBC expression based on sample type . (B) CCND1 expression based on sample type. (C) ESR1 expression base on sample types. (D) ACTB expression base on sample types. (E) TP53 expression base on sample types. (F) TP53 expression based on tumor grade. (G) TP53 expression based on nodal metastasis status. (H) MYC expression based on sample type. (I) HSP90AA1 expression based on sample type. (J) PTEN expression based on sample type. (K) CASP3 expression based on sample type. (L) CTNNB1 expression based on sample type. (M) AKT1 expression based on sample type. (N) MAPK3 expression based on sample type. (O) CDC42 expression based on sample type. (P) MAPK1 expression based on sample type. * P< 0.05 ; ** $\mathrm{P}<0.01 ; * * * \mathrm{P}<0.001$; **** $\mathrm{P}<0.0001$. 


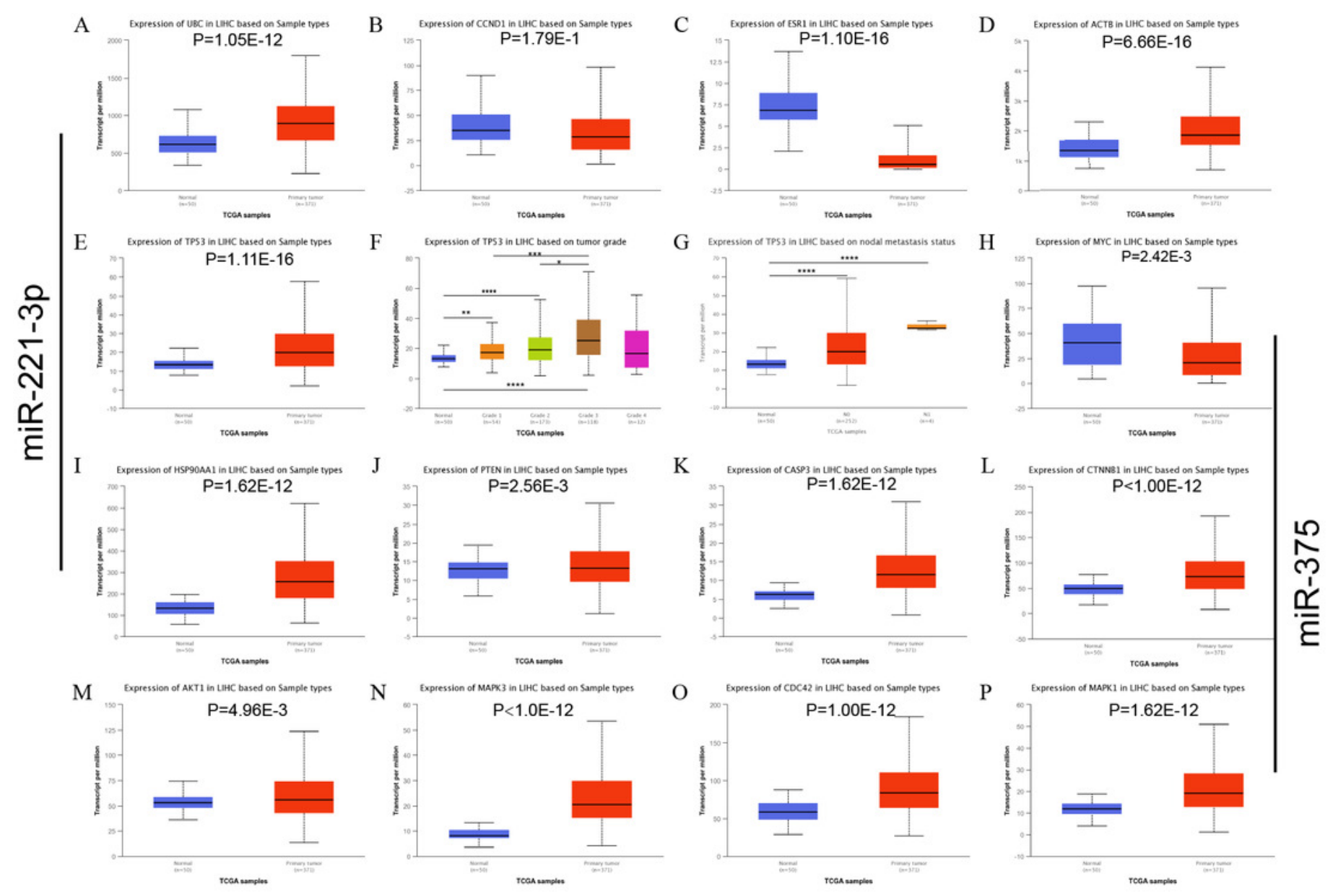




\section{Figure 4}

Figure 4 The expression and prognostic roles of miR-221-3p and miR-375 in HCC.

The expression level of miR-221-3p (A) and miR-375 (D) in three HCC cell lines (HepG2, HepG2.2.15, MHCC-LM3) and one normal liver cell line (HL7702) detected by qRT-PCR. MiR-221-3p and miR-375 were significantly upregulated and downregulated in all three HCC cell lines, respectively, compared to HL7702 cells. The expression level of miR-221-3p (B) and miR-375 (E) in 20 clinical HCC samples vs matched para-tumor tissues detected by qRTPCR. MiR-221-3p and miR-375 were, respectively, upregulated and downregulated in 20 HCC tissue samples compared to their para-tumor tissues. The mean expression level of miR-221-3p (C) and miR-375 (F) in multiple clinical HCC samples vs. normal tissues, as assessed by OncomiR online analysis. The mean expression level of miR-221-3p and miR-375 in multiple clinical HCC samples was much higher and lower, respectively, than that of normal tissues. Kaplan-Meier survival curve of miR-221-3p (G) and miR-375 (H) based on percentile cutoff value $50 \%$ in HCC, as determined by OncomiR online analysis. high miR-221-3p expression and low miR-375 expression tended to be correlated with poor overall survival. However, this association was not statistically significant $(P=0.06034$ for miR-221-3p and $P=0.3215$ for miR-375). The cohort labeled as high-risk (red line) represents levels of miRNA expression above the average level; the cohort labeled as low-risk (blue line) represents miRNA expression levels below the average level. One-way ANOVA analysis was used to compare the miRNA expression between four cell lines. The two-independent $t$-test was used to compare miRNAs expression between para-tumor and tumor tissues. $* \mathrm{P}<0.05$; ** $\mathrm{P}<0.01 ;{ }^{* * *} \mathrm{P}<0.001 ;{ }^{* * * *} \mathrm{P}<0.0001$. 


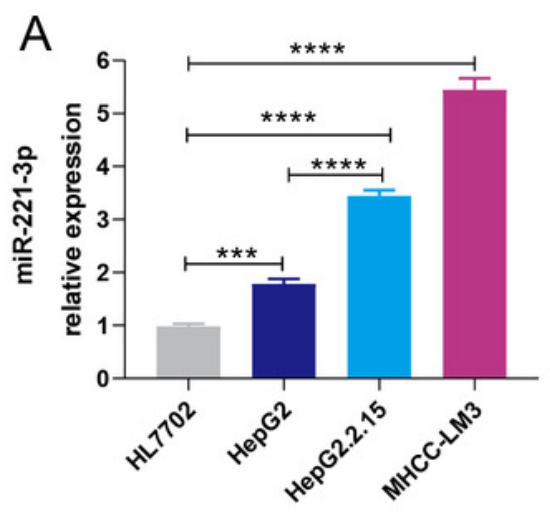

D
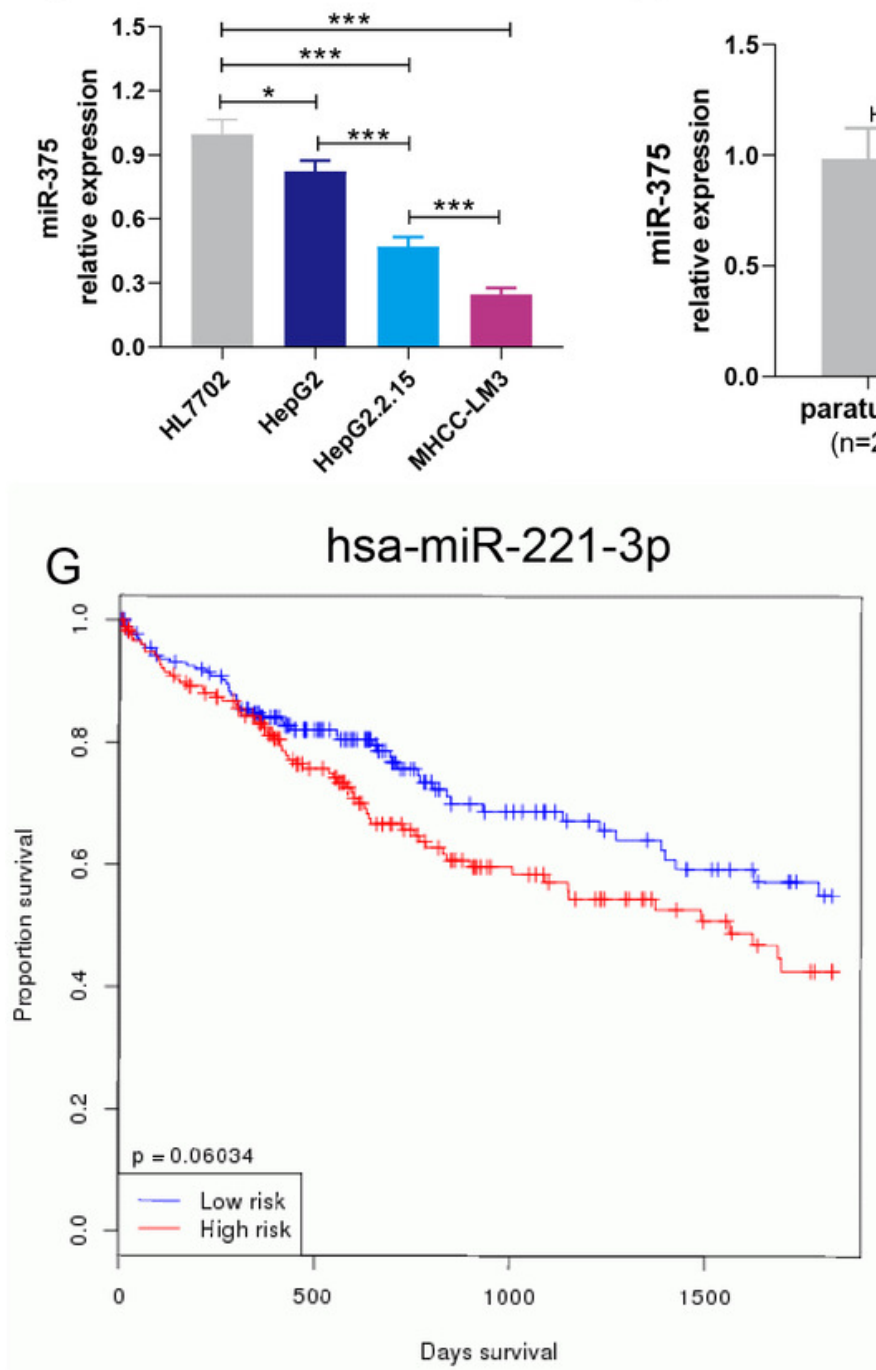

OncomiR
B

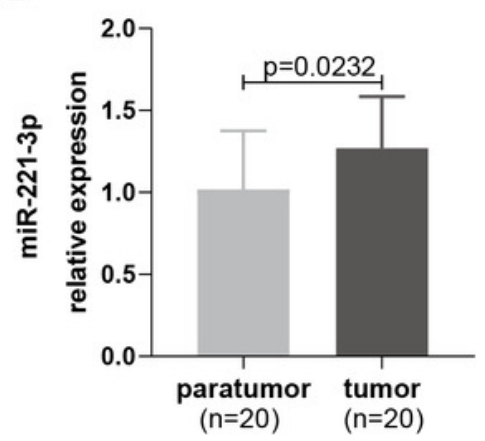

E

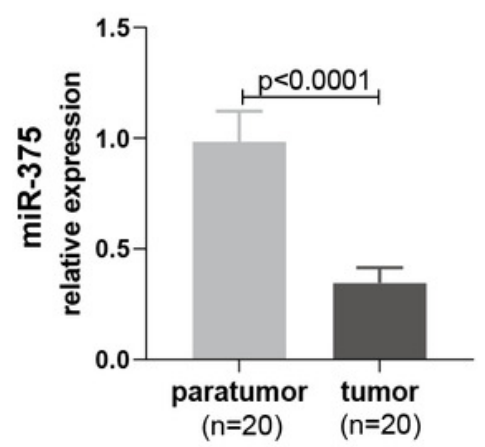

C

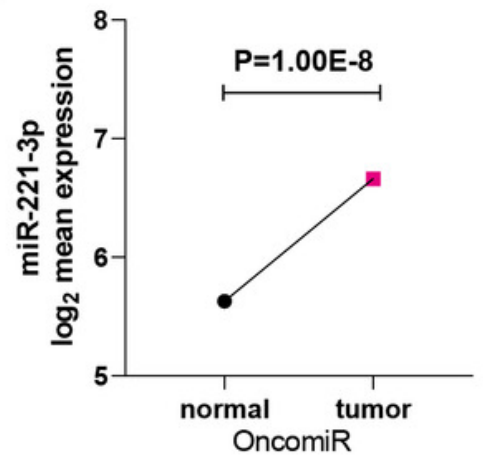

F

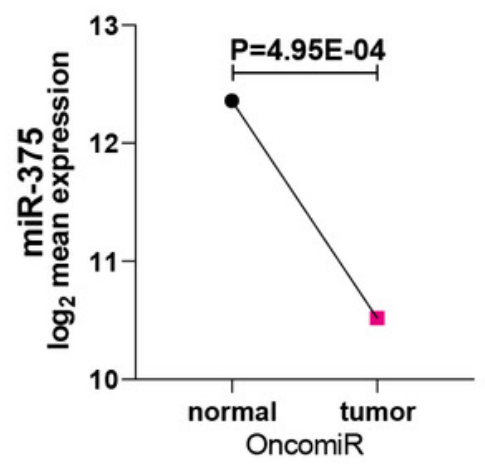

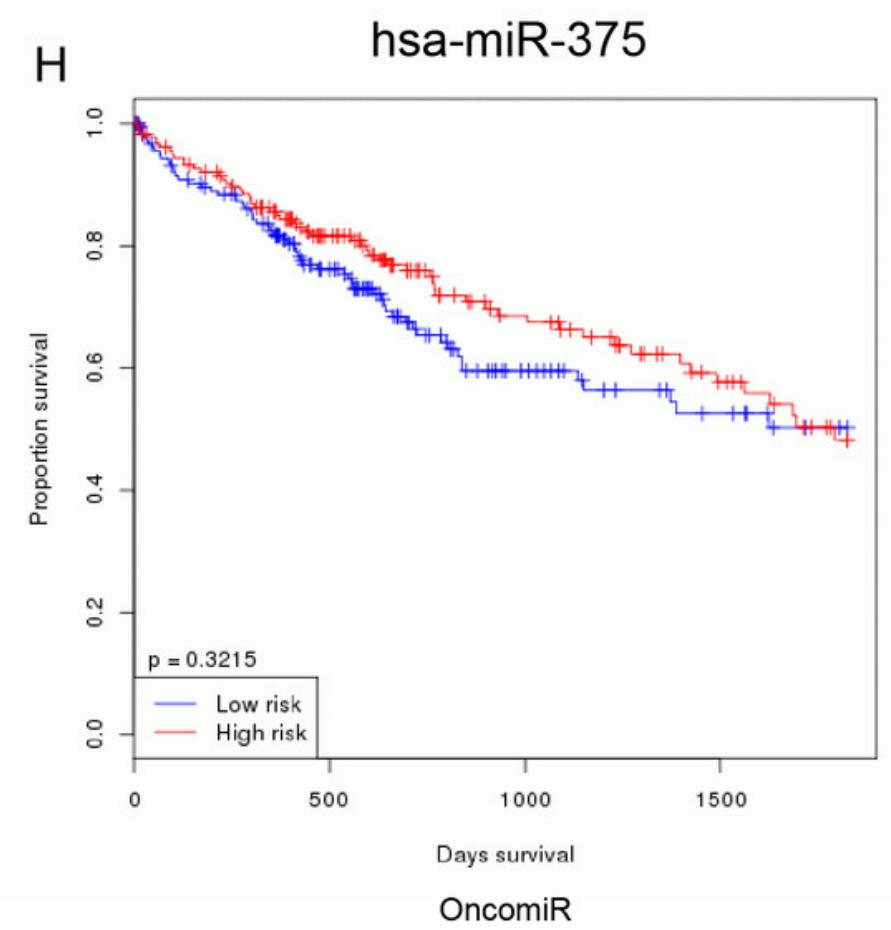




\section{Figure 5}

Figure 5 Overexpression of miR-221-3p and miR-375 affect cell proliferation, HBV-DNA replication, and TP53 expression in HCC cell lines.

(A) The relative expression level of miR-221-3p/NC and miR-375/NC after transfection with NC, miR-221-3p mimics, and miR-375 mimics (at the final concentrations of $50 \mathrm{nM}$ or $100 \mathrm{nM}$ ) in HepG2 cells. The miRNA mimics (miR-211-3p and miR-375) significantly increased the expression of the corresponding miRNAs at both concentrations. (B) The overexpression of miR-221-3p and miR-375 in HepG2 cells enhanced and reduced, respectively, cell proliferation, as assessed by MTT analysis. (C) The overexpression of miR-221-3p and miR-375 in Hepg2.2.15 cells increased and decreased, respectively, the level HBV-DNA, as demonstrated by qRT-PCR. (D) The overexpression of miR-221-3p and miR-375 downregulated and upregulated, respectively, TP53 expression, as assessed by western blotting. (E) and (F) Analysis of the co-expression of miR-211-3p/TP53 (E) and miR-375/TP53 $(F)$ in HCC clinical tissue samples using starBase online software. The expression of both miR-221-3p and miR-375 was positively correlated with TP53 expression ( $r=0.313$ and Pvalue $=7.16 \mathrm{E}-10$ for miR-221-3p; $r=0.036$ and P-value $=0.495$ for miR-375). The twoindependent $t$-test was used to compare the difference of miRNA relative expression, MTT, and HBV-DNA. ${ }^{* *} \mathrm{P}<0.01 ; * * \mathrm{P}<0.001$. 


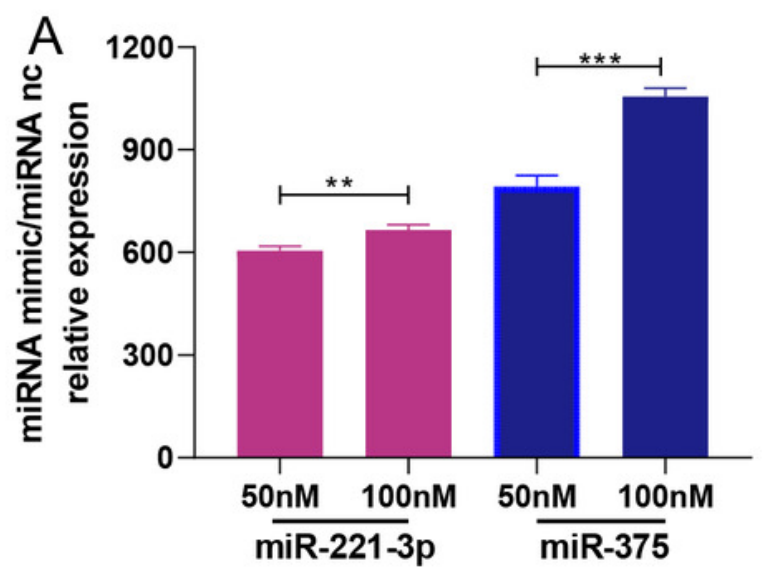

B
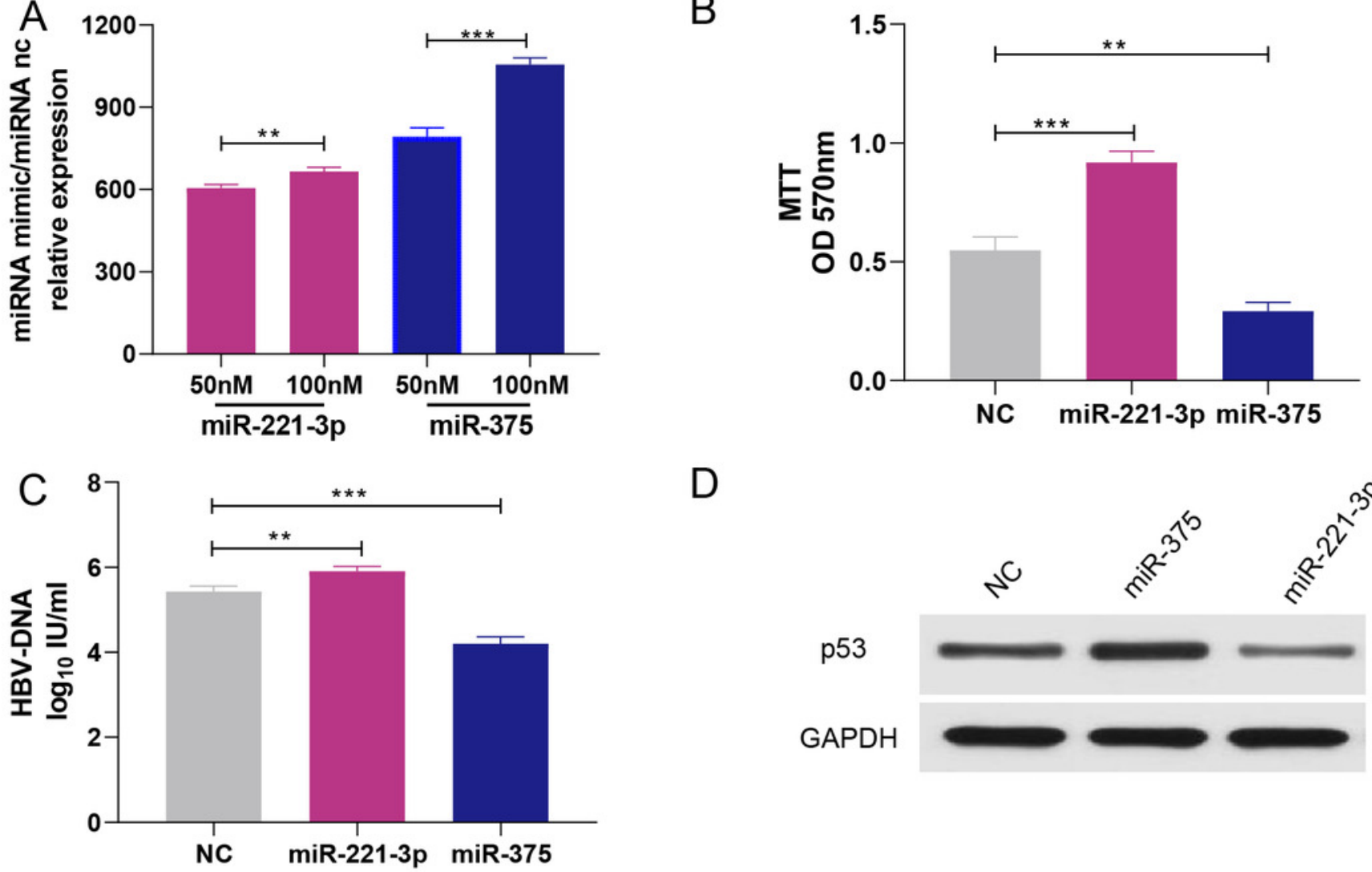

D

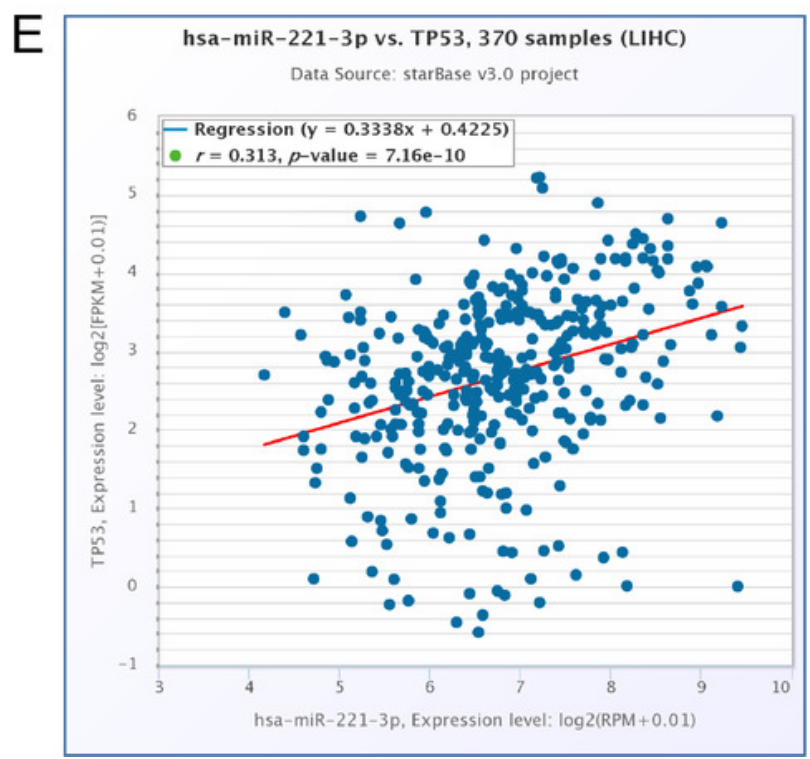

$\mathrm{F}$

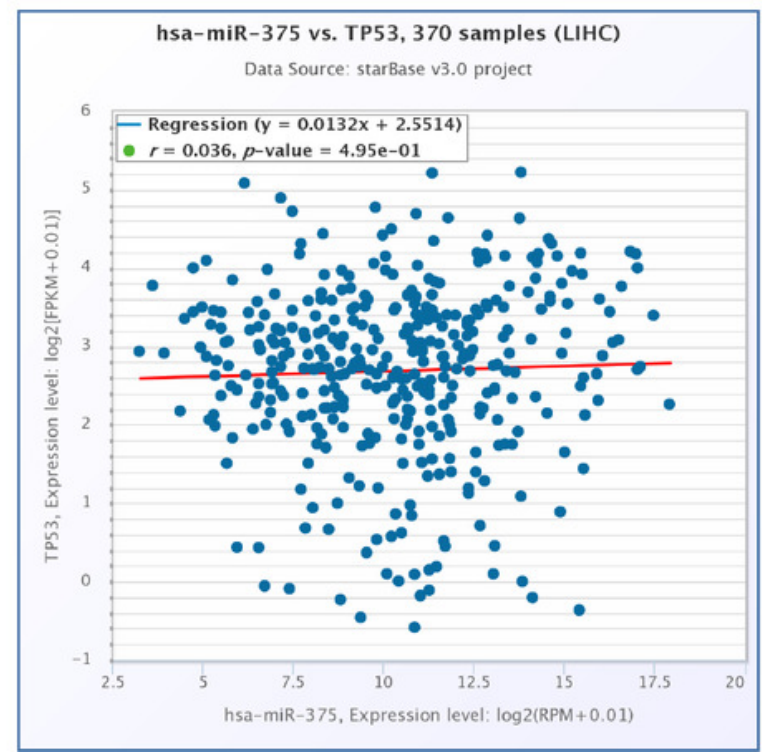




\section{Figure 6}

Figure 6 Overexpression of miR-221-3p and miR-375 enhanced and suppressed, respectively, the migration and invasion of HepG2 cell lines.

(A) The wound healing assay was performed after transfection with NC, miR-221-3p mimic (50 nM), or miR-375 mimic ( $50 \mathrm{nM})$, and an additional $24 \mathrm{~h}$ of culture (picture magnification: 100x; black bar: $100 \mu \mathrm{m}) ;$ HepG2 transfected with miR-221-3p and miR-375 mimics exhibited higher and lower migration ability compared to NC-transfected cells, respectively. (B) Quantification from A. (C) The transwell assay was performed after transfection with NC, miR-221-3p mimic ( $50 \mathrm{nM}$ ) or miR-375 mimic ( $50 \mathrm{nM}$ ) and an additional $24 \mathrm{~h}$ of culture (picture magnification: 200x; black bar: $50 \mu \mathrm{m}$ ). HepG2 transfected with miR-221-3p miR-375 mimics were more and less invasive compared to those transfected with NC, respectively. (D) Quantification from $C$. The two-independent $t$-test was used to compare the difference of wound healing rate and cell number. $* * P<0.01$; $* * * P<0.001$. 


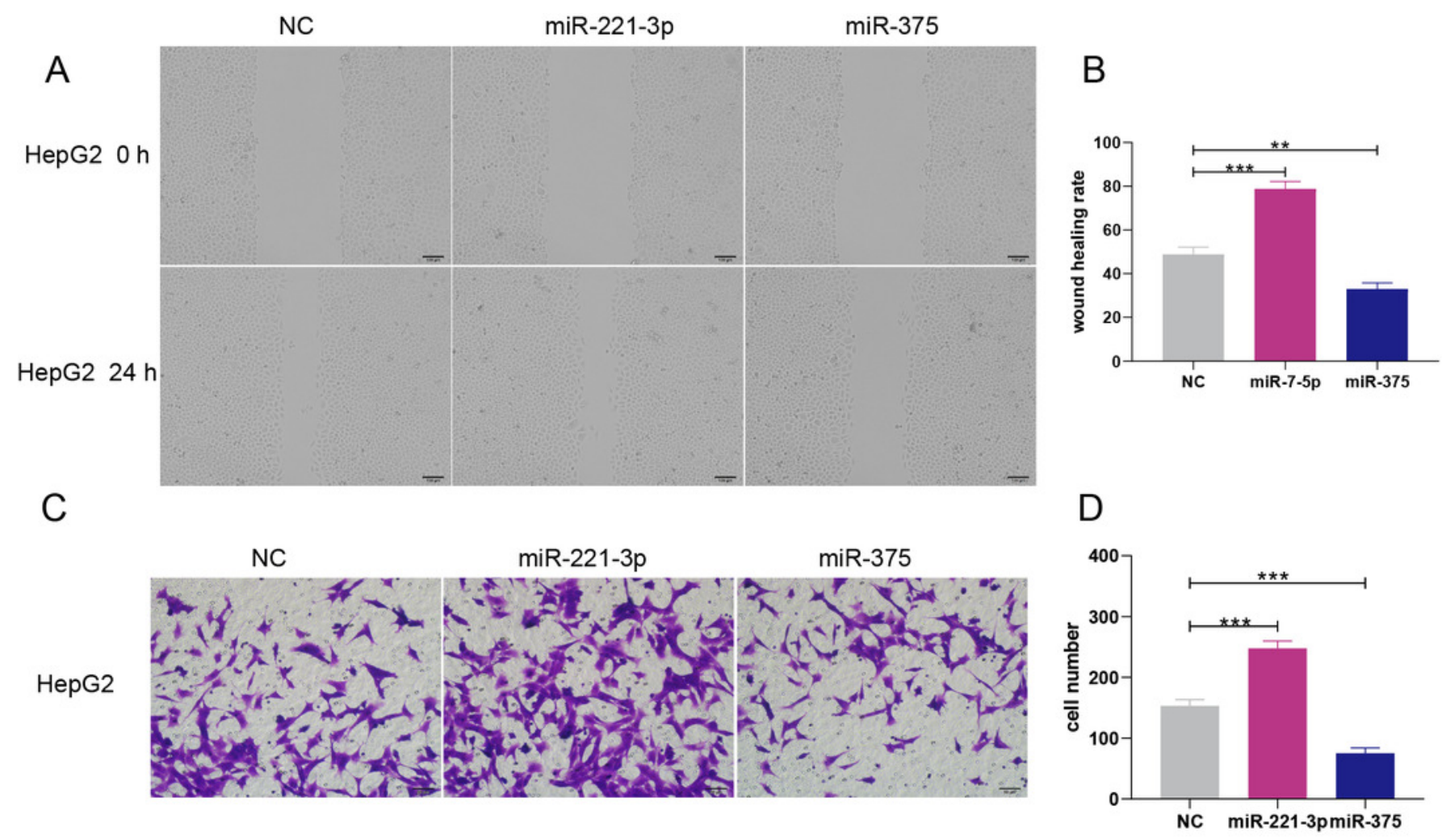




\section{Table $\mathbf{1}$ (on next page)}

Table 1 The top ten upregulated miRNAs between $\mathrm{HCC}$ and matched adjacent tumorfree tissues ranked by $\left|\log _{2} \mathrm{FC}\right|$. 
1 Table 1 The top ten upregulated miRNAs between HCC and matched adjacent tumor-free tissues 2 ranked by $\log _{2} \mathrm{FC}$.

\begin{tabular}{lllll}
\hline ID & $\log _{2} \mathrm{FC}$ & P-value & $\mathrm{t}$ & $\mathrm{B}$ \\
\hline hsa-miR-221-3p & 3.75 & $1.27 \mathrm{E}-03$ & 4.10 & -0.73 \\
hsa-miR-222-3p & 3.57 & $1.70 \mathrm{E}-03$ & 3.95 & -0.99 \\
hsa-miR-18b-5p & 3.41 & $1.12 \mathrm{E}-02$ & 2.96 & -2.70 \\
hsa-miR-500a-3p & 2.80 & $2.57 \mathrm{E}-02$ & 2.52 & -3.44 \\
hsa-miR-196b-5p & 2.80 & $1.62 \mathrm{E}-02$ & 2.77 & -3.03 \\
hsa-miR-7-5p & 2.60 & $1.21 \mathrm{E}-02$ & 2.92 & -2.77 \\
hsa-miR-6516-3p & 2.55 & $2.39 \mathrm{E}-02$ & 2.56 & -3.38 \\
hsa-miR-34a-3p & 2.46 & $1.72 \mathrm{E}-02$ & 2.73 & -3.09 \\
hsa-miR-362-3p & 2.13 & $1.08 \mathrm{E}-02$ & 2.97 & -2.67 \\
hsa-miR-339-3p & 2.02 & $1.35 \mathrm{E}-02$ & 2.86 & -2.87 \\
\hline
\end{tabular}

3

4

5

6

7

8 


\section{Table 2 (on next page)}

Table 2 The top ten downregulated miRNAs between HCC and matched adjacent tumorfree tissues ranked by $\left|\log _{2} \mathrm{FC}\right|$. 
1 Table 2 The top ten downregulated miRNAs between HCC and matched adjacent tumor-free 2 tissues ranked by $\log _{2} \mathrm{FC}$.

\begin{tabular}{lllll}
\hline ID & $\log _{2} \mathrm{FC}$ & $P$ value & $\mathrm{t}$ & $\mathrm{B}$ \\
\hline hsa-miR-375 & -6.41 & $1.51 \mathrm{E}-07$ & -10.22 & 6.72 \\
hsa-miR-214-3p & -4.90 & $1.94 \mathrm{E}-03$ & -3.88 & -1.11 \\
hsa-miR-378d & -4.20 & $2.38 \mathrm{E}-03$ & -3.77 & -1.30 \\
hsa-miR-486-5p & -4.13 & $1.37 \mathrm{E}-03$ & -4.06 & -0.80 \\
hsa-miR-199a-5p & -3.86 & $3.51 \mathrm{E}-03$ & -3.56 & -1.65 \\
hsa-miR-8089 & -3.54 & $1.34 \mathrm{E}-03$ & -4.08 & -0.77 \\
hsa-miR-338-3p & -3.28 & $2.91 \mathrm{E}-03$ & -3.66 & -1.48 \\
hsa-miR-7845-5p & -3.20 & $1.98 \mathrm{E}-03$ & -3.87 & -1.13 \\
hsa-miR-199a-3p & -2.95 & $4.74 \mathrm{E}-04$ & -4.64 & 0.16 \\
hsa-miR-10a-5p & -2.13 & $1.23 \mathrm{E}-03$ & -4.12 & -0.70 \\
\hline
\end{tabular}

3

4

5

6

7 


\section{Table 3(on next page)}

Table 3 The top ten hub genes of target gene sets predicted by three upregulated and downregulated miRNAs ranked by degrees. 
1 Table 3. The top ten target hub genes of three upregulated and downregulated DE-miRNAs 2 ranked by degrees.

\begin{tabular}{|c|c|c|c|c|c|}
\hline \multicolumn{3}{|c|}{ upregulated DE-miRNAs } & \multicolumn{3}{|c|}{ downregulated DE-miRNAs } \\
\hline Rank & Name & Score & Rank & Name & Score \\
\hline 1 & TP53 & 172 & 1 & TP53 & 151 \\
\hline 2 & $M Y C$ & 128 & 2 & AKT1 & 141 \\
\hline 3 & HSP90AA1 & 108 & 3 & MYC & 107 \\
\hline 3 & $U B C$ & 105 & 4 & PTEN & 90 \\
\hline 5 & PTEN & 104 & 5 & MAPK3 & 87 \\
\hline 6 & CCND1 & 91 & 6 & HSP90AA1 & 84 \\
\hline 7 & CASP3 & 82 & 7 & CTNNB1 & 83 \\
\hline 8 & CTNNB1 & 82 & 8 & $C D C 42$ & 82 \\
\hline 9 & ESR1 & 81 & 9 & MAPK1 & 77 \\
\hline 10 & $A C T B$ & 79 & 10 & CASP3 & 76 \\
\hline
\end{tabular}

3 